\title{
Dialectical behavior therapy as a tool for reducing addictive behaviors and attitudes: an adolescent
} group review

\begin{abstract}
a) Self-destructive behaviors and a propensity for relapse
b) Identity disturbances and low self esteem
c) Relationship conflict and social skills difficulties and
d) Dichotomous thinking and emotion dysregulation

Dialectical Behavior Therapy (DBT) has been proven to be successful for reducing symptoms related to Borderline Personality Disorder. ${ }^{1,2}$ However, because of the gap that exists between mental health and substance abuse treatment approaches, little research has been done to show the effects of DBT approaches on addictive thinking and behaviors. In addition, only recently have researchers begun applying DBT interventions with a teenage population. ${ }^{3}$ Using self-report methods, the authors examine the use of a modified DBT approach with 13 participants from ages 14-26, exploring the effects of a DBT group modality on symptoms commonly found in individuals with substance use disorders (SUDs). Preliminary findings resulted in an overall mean score of 3.24 for 14 items corresponding to SUD symptomology, suggesting that DBT interventions helped to reduce.

Future studies are needed using larger sample sizes, informant reports, and a sample of individuals with a primary diagnosis of SUDs, to gain confidence in these findings.

Keywords: dialectical behavior therapy, addictive behaviors, adolescent, substance use disorders, DBT
Volume I Issue I - 2015

\author{
Suzanne Robison,' Nicole Luczakowsky² \\ 'Graduate School of Education, University of Pennsylvania, USA \\ ${ }^{2}$ Community and Trauma Counseling, Philadelphia University,
} USA

Correspondence: Suzanne Robison, Graduate School of Education, University of Pennsylvania, I 476 West Main Street Lansdale, PA, USA, Tel 610-247-6782, Fax 215-717-4646, Email therapy@suzannerobison.com

Received: May 20, 2015 | Published: June 18, 2015
Abbreviations: DBT, dialectical behavior therapy; BPD, borderline personality disorder; SUD, substance use disorder; DSM-V, diagnostic and statistical manual of mental disorders, fifth edition; APA, american psychiatric association; COA, children of alcoholics

\section{Introduction}

DBT has been proven to be a successful method of treatment for minimizing many symptoms of BPD. ${ }^{4}$ Because BPD is typically an adult diagnosis, there is less research to support the use of DBT with minors. Yet there are many times that it is appropriate to diagnose an adolescent with this disorder, leading clinicians and family members to question what is the most effective care for a younger person. Because of the efficacy of DBT with adults, many researchers and clinicians have begun using the model, or a modified version of it, with a younger population, resulting in promising outcomes. ${ }^{3,5}$ As is the case with the application of DBT with an adolescent population, utilizing DBT with a SUD population is a natural extension of its use with BPD clients. While substance-using clients differ in many ways from clients presenting with BPD, there are several overlapping behaviors, attitudes, and treatment needs that cannot be denied.

\section{Symptom commonalities between BPD and (SUDs)}

BPD and SUDs are often comorbid, ${ }^{6}$ making it difficult to determine primary and secondary diagnoses. While many individuals with BPD act out with substances, there is also a high comorbidity between BPD and trauma, ${ }^{7,8}$ with substance use and other selfdestructive behaviors developing as coping skills for the trauma. ${ }^{9,10}$ The complicated clusters of behaviors and symptoms make differential diagnosis just as complicated, reducing the value of using a diagnosis to guide treatment planning. The danger of incorrectly perceiving certain diagnoses as comorbid, particularly personality disorders which are found to have a significantly high comorbidity with many other disorders, ${ }^{11,12}$ have resulted in Maj ${ }^{13}$ suggesting that the term "comorbid" be avoided altogether. Selecting treatment interventions based on symptom presentation, rather than diagnosis, may therefore yield more positive results for the individual. In taking this approach, the similarities between SUD and BPD symptomology are striking.

In 1992 the American Medical Association defined alcoholism as a chronic, progressive disease that is influenced by genetic, psychosocial and environmental factors, with the most notable symptom of the disease being denial. ${ }^{14}$ Similarly, in 2013 the APA ${ }^{6}$ describes personality disorders as being pervasive and chronic, and as having multiple influences on epidemiology. Furthermore, Millon et al. ${ }^{15}$ note that personality disorders have egosyntonic symptoms, resulting in a significant amount of denial. In addition to self-destructive behaviors and a propensity to relapse, identity disturbances and low self esteem, relationship conflict and social skills deficits, and dichotomous thinking and emotion dysregulation are among the symptoms that straddle both BPD and SUDs. ${ }^{6,16}$

\section{Self destructive behaviors and a propensity to relapse}

The Diagnostic and Statistical Manual of Mental DisordersFifth Edition ${ }^{6}$ list's suicidal or parasuicidal behaviors, as well as impulsivity, as potential symptoms of BPD. These symptoms often lead to complications related to substance use, eating disordered behaviors, or other risky acts. ${ }^{6,9,17}$ SUDs, according to criteria listed 
within the DSM-V, involve compulsive use and obsessive thinking related to substances. ${ }^{6}$ Similarly, obsessive thinking has been found to be common for individuals with BPD. ${ }^{18}$ While many individuals with BPD engage in self harming behaviors such as cutting or burning, ${ }^{1,6}$ the obsessive and compulsive nature of these behaviors, as well as the tendency toward relapse, result in presentations and treatment needs that are similar to those with SUDs.

\section{Identity disturbance and low self esteem}

Low self-efficacy and low self-esteem are risk factors for substance use, ${ }^{19,20}$ yet are often reported by individuals with BPD as well. ${ }^{8}$ While identity disturbance is a symptom often experienced by individuals with $\mathrm{BPD},{ }^{6}$ adolescents and young adults may be appropriately struggling with identity formation, which may become exacerbated by peer conflict or pressure to identify a career choice. ${ }^{6}$ While forming an identity is an appropriate developmental milestone, Santrock ${ }^{21}$ emphasizes the importance of effective parenting on identity formation, noting that certain parenting styles may interfere with an individual's ability to establish a healthy identity during critical periods of development. While it is important not to pathologize normal developmental milestones, recognizing and treating potential identity problems earlier in development is likely to minimize negative effects later in life.

The struggle to fit in and establish a stable sense of self that is seen in individuals with BPD is also a common problem for individuals with SUDs. Chronic feeling of emptiness, ${ }^{6}$ another symptom of $\mathrm{BPD}$, may motivate excessive use of substances or other dangerous behaviors, as an effort to externally fill an internal need. It is also likely however, that individuals with BPD, in their efforts to establish their identities, may associate themselves with other individuals engaging in self-destructive behaviors, such as substance use. It is appropriate for clinicians to make this a significant focus of treatment, as substance users who identify themselves as part of the addictive subculture have poorer recovery outcomes ${ }^{22}$ while identifying one's self as a cutter is correlated with a more severe level of pathology. ${ }^{23}$

\section{Relationship conflict and social skills deficits}

One of the most important recovery goals for maintaining drug and alcohol sobriety is adopting a sober lifestyle, while abandoning connections with drug using peers. ${ }^{24}$ Best et al. ${ }^{25}$ noted that an increased number of meaningful interactions with supportive individuals (especially recovering individuals) were related to better recovery outcomes. Capuzzi \& Stauffer ${ }^{26}$ note that requirements for effective SUD prevention programs include social skills training, parental involvement, peers as educators and inclusion of members of the community. The success of 12 step fellowships in maintaining long-term sobriety ${ }^{27,28}$ is a testament to the importance of socialization and positive social interactions.

Similarly, individuals with BPD experience, repeated chaotic interpersonal relationships often exacerbated by intense fear of abandonment. ${ }^{6}$ While healthy relationships and support can be instrumental in facilitating change for individuals with either BPD or SUDs, ${ }^{29}$ the lack of insight and high level of dependency that often accompany these disorders can be very damaging to relationships; causing a reduction in an individual's much needed support system.

\section{Dichotomous thinking and emotion dysregulation}

Substances act directly on the limbic system, which can interfere with ability to regulate emotions and control behaviors, ${ }^{30}$ contributing to the dichotomous thinking patterns often seen in addicted individuals. ${ }^{26}$ Substance-using adolescents have been found to have more psychological distress, coupled with fewer of the problemsolving and coping skills needed to help regulate this distress. ${ }^{31-33}$ Emotional problems, personality disorders and suicidality have also been found to be associated with poly drug use in adolescence. ${ }^{34-36}$

According to the APA, ${ }^{6}$ symptoms of dichotomous thinking and difficulty regulating emotions are included as BPD criteria. Linehan $^{8}$ presents dialectical dilemmas in her DBT treatment model that describe many of the extremes that individuals with BPD vacillate between, as well as some of the treatment implications of these dilemmas. According to Linehan, emotional vulnerability (the predisposition to be sensitive and reactive to emotions) can greatly interfere with functioning; however, the tendency these individuals have to invalidate their own emotional states can significantly worsen these symptoms. Many invalidating statements may have been internalized from childhood, as the combination of an invalidating environment and emotional vulnerability has been shown to increase the risk of developing BPD. ${ }^{37}$ While invalidating environments may stem from a multitude of problem behaviors or diagnoses, there are again many striking similarities between symptoms experienced by individuals with BPD and those experienced by children of alcoholics (COA); further substantiating the overlap between SUD-related factors and BPD (Table 1).

Table I Comparison of Symptoms Associated with COA and Individuals with $\mathrm{BPD}^{26}$

\begin{tabular}{lll}
\hline Symptom & COA & BPD \\
\hline Invalidation of one's own feelings & $\mathrm{X}$ & $\mathrm{X}$ \\
Low self worth & $\mathrm{X}$ & $\mathrm{X}$ \\
Mistrust of others & $\mathrm{X}$ & $\mathrm{X}$ \\
Conflictual relationships & $\mathrm{X}$ & $\mathrm{X}$ \\
Dichotomous thinking & $\mathrm{X}$ & $\mathrm{X}$ \\
Feeling of unreality & $\mathrm{X}$ & $\mathrm{X}$ \\
Cognitive distortions & $\mathrm{X}$ & $\mathrm{X}$ \\
\hline
\end{tabular}

COA, children of alcoholics; BPD, borderline personality disorder

Many of the symptoms commonly experienced by individuals with BPD or SUDs can be organized into the above 4 categories. Depending upon their severity, these symptoms can greatly interfere with the functioning and overall quality of life of individuals no matter what their diagnoses. This, coupled with the high-risk and often lethal nature of both of these disorders, makes it critical to increase the body of research around treatment interventions and their efficacy. This study offers preliminary data to encourage and support clinicians in selecting DBT treatment interventions for individuals with SUDs, which in turn may open the door to further, more in-depth analysis of the effectiveness of these interventions.

\section{Materials and methods}

1 male and 12 female respondents ranging in age from 1426 participated in this study. DBT group members ranged from newcomers attending for duration of only 4 weeks at the time of responding, to more senior members attending for over 2 years. 3 of the participants had discontinued group in the past and resumed again in an effort to re-learn or increase application of skills learned. 
Individuals providing responses all met the criteria for BPD, and had a wide variety of concurrent diagnoses, while 1 participant had a concurrent SUD diagnosis.

Respondents were given a 20-item group rating form used to evaluate the skills learned in DBT and group process, using a 4-point Likert scale. All group members were asked to complete the form before the start of group, with data being collected from two different groups on 2 separate nights. Group content was the same for both groups and the same leader ran both groups. The group followed the manualized structure of the DBT model, ${ }^{8}$ with some modifications intended to make the content more age appropriate, and to expand on content to meet the needs of the individuals (such as trauma recovery content, 12-step philosophy, education about family dynamics, etc). Participation was voluntary and responses were kept anonymous, with all group members contributing feedback with the exception of 2 group members who were absent on the night of data collection.

Review of group rating forms resulted in identification of the 4 key areas of study that were determined by the authors to be related to addictive symptomology: self destructive behaviors and a propensity to relapse, identity disturbances and low self-esteem, relationship conflict and social skills deficits, and dichotomous thinking and emotion dysregulation. Item selection resulted in 14 items being identified as corresponding to these 4 areas. Mean responses were calculated in an effort to determine group member's perceptions of the usefulness of a DBT approach in reducing these problematic attitudes and behaviors. The percentages and range of responses, as well as the standard deviation were calculated to allow for increased understanding of the data set.

\section{Results and discussion}

While DBT has strong support in the research with regard to its effectiveness in reducing BPD-related symptoms, ${ }^{1,2}$ as do modified DBT programs for adolescents, ${ }^{38,39}$ more research is needed with regard to the effectiveness of these interventions with SUDs. ${ }^{40}$ DBT was created specifically for BPD and, while arguments could be made that SUDS are distinctly different conditions than personality disorders, there are multiple overlapping symptoms among the disorders that cannot be denied. Further, there are striking similarities between BPD symptomology and problems associated with COA (Table 1), allowing us to draw inferences about the usefulness of DBT with individuals with addictive family patterns. Results of the selfreport data provided in this study indicate that a DBT or a modified DBT approach may be very useful in reducing addictive behaviors and attitudes.

\section{Preliminary results}

$85 \%$ of the respondents in this study reported DBT to be very helpful, or extremely helpful in reducing all 14 problem items related to the 4 areas identified as common to addictive symptomology. $2 \%$ of the responses given indicated that DBT skills were not helpful at all for the individual items inquired about, with $38 \%$ of the responses indicating these skills to be extremely helpful in reducing these behaviors and attitudes. Using a 4-point Likert Scale, the mean scores for each of the corresponding categories ranged from 3 to 3.36 with an overall mean of 3.24 and a standard deviation of 0.16 (Figure 1). The overall mean indicates a perception of DBT skills being very helpful in reducing symptomology relating to BPD and SUDs. The standard deviation indicates that there is very little variability among the scores.

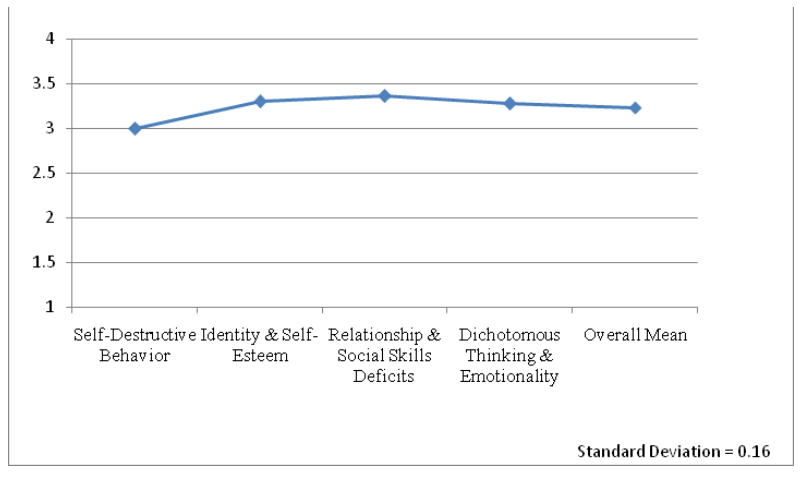

Figure I Mean distribution across 4 categories of DBT group rating forms.

\section{Limitations}

While this study reveals a modified DBT approach to be perceived as very beneficial to reducing symptoms related to addictive symptomology and BPD, this is only a preliminary investigation requiring further study and statistical analyses. In addition, although research supports many of these symptoms as related to addictive diseases, this study was done with a sample of individuals with BPD, not SUDs, so a sample of individuals with primary SUDs may not yield similar results. Furthermore, this is a very small sample size, using a measure that does not have established validity or reliability, and measuring responses within an open group format, which may have skewed the data. Although individual responses were kept anonymous, there may also have been some response bias, since individuals were asked to fill out their group rating forms together. This method of data collection may have increased the risk of, or the perception of risk of others seeing personal responses. Finally, descriptive rather than inferential statistical measures were used in this study, weakening the results.

\section{Future directions}

While self-report measures are a good start to exploring the effectiveness of a modified DBT approach in reducing SUD symptoms, further data collection is needed to increase the validity of these self-reports. Gathering informant data would be beneficial, in order to increase the validity of these responses. Using a larger sample size, inferential statistics to analyze the data, and including multiple valid and reliable measures would increase construct validity to allow for generalizability to the larger population. Finally, including individuals with a primary diagnosis of a SUD is necessary to establish the effectiveness of DBT with the population as a whole.

\section{Conclusion}

There are endless concerns about how SUDs affect the individual, the family, and the larger community. These are among the hardest disorders to treat, with relapse to substances being a reality for at least $50 \%$ of individuals, ${ }^{41}$ and relapse to addictive behaviors and attitudes being far more likely. Similarly, personality disorders are notoriously difficult to treat, and often present comorbidly, ${ }^{6,15}$ resulting in much bias and misunderstanding by treatment providers, and by the general public. The introduction of DBT in the early 90 ' $\mathrm{s}^{1}$ changed the way of thinking about BPD, and it is now listed in the National Registry of Evidence Based Programs and Practices. ${ }^{4}$ Research utilizing a DBT model with a SUD population is lacking however, ${ }^{40}$ resulting in alternative treatment models being favored for use. ${ }^{42}$ Because of the significant commonalities between BPD and SUD symptoms, 
it is reasonable to assume that the application of DBT with a SUD population would be effective as well. While this study includes a small sample size, preliminary data analysis, and self-report measures, further exploration of the effectiveness of DBT with a SUD population is warranted; due to the positive reports of symptom reduction identified by participants in this study.

\section{Acknowledgements}

The authors would like to acknowledge the hard work and dedication that the members of these DBT groups put forth. Symptom reduction is, more than anything else, primarily the result of this type of commitment and motivation for change. The authors are inspired by the incredible courage and tenacity that the amazing young men and women participating in these groups consistently display.

\section{Conflict of interest}

This study was done in for a profit, private practice location, not affiliated with Universities where the authors are considered to be students or faculty.

\section{References}

1. Linehan MM, Heard HL, Armstrong HE. Naturalistic follow-up of a behavioral treatment for chronically parasuicidal borderline patients. Arch Gen Psychiatry. 1993;50(9):971-974.

2. van den Bosch LM, Koeter MW, Stijnen T, et al. Sustained efficacy of dialectical behavioral therapy for borderline personality disorder. Behav Res Ther. 2005;43(9):1231-1241.

3. Miller AL, Rathus JH, Linehan MM. Dialectical Behavior Therapy with Suicidal Adolescents. USA: Guilford Press; 2007. p. 1-346.

4. Fleischhaker C, Bohme R, Sixt B, et al. Dialectical Behavioral Therapy for Adolescents (DBT-A): a clinical Trial for Patients with suicida and self-injurious Behavior and Borderline Symptoms with a one-year Follow-up. Child Adolesc Psychiatry Ment Health. 2011;5(1):3

5. American Psychiatric Association. Diagnostic and statistical manual of mental disorders. 5th ed. Author, Washington DC, USA, 2013. p. 1-991.

6. Favazza AR. Bodies under siege: self-mutilation, Non-Suicidal Self-Injury, and body modification in culture and psychiatry. 3rd ed. Baltimore, MD, US: Johns Hopkins University Press; 2011. p. 1-352.

7. Linehan MM. Cognitive-behavioral treatment of borderline personality disorder. New York: Guilford Press; 1993. p. 1-558.

8. Favazza AR, Conterio K. Female habitual self-mutilators. Acta Psychiatr Scand. 1989;7(9):238-289.

9. Van der Kolk BA. Developmental trauma disorder: Towards a rational diagnosis for children with complex trauma histories. Psychiatry Annals. 2005;35(5):401-408.

10. First MB. Mutually Exclusive versus co-occurring diagnostic categories: the challenge of diagnostic comorbidity. Psychopathology. 2005;38(4):206-210.

11. Grasmo I. High personality disorder comorbidity rates identified in anxiety. Medical Research News. 2012.

12. Maj M. Psychiatric comorbidity: An artefact of current diagnostic systems? Br J Psychiatry. 2005;186(3):182-184

13. Morse RM, Flavin DK. The definition of alcoholism. The Join Committee of the National Council on Alcoholism and Drug Dependence and the American Society of Addiction Medicine to Study the Definition and Criteria for the Diagnosis of Alcoholism. JAMA. 1992;268(8):10121014 .
14. Millon T, Millon CM, Meagher S, et al. Personality disorders in Modern life. 2nd ed. USA: John Wiley \& Sons; 2004. p. 1-664.

15. Frings D, Albery IP. The Social Identity Model of Cessation Maintenance: formulation and initial evidence. Addict Behav. 2015;44:35-42.

16. Darche MA. Psychological factors differentiating self-mutilating and non-self-mutilating adolescent inpatient females. Psychiatric Hospital. 1990;21(1):31-35.

17. Sauer-Zavala SE, Geiger PJ, Baer RA. The effect of anger rumination in the relationship between borderline personality disorder symptoms and precursors. J Pers Disord. 2013;27(4):465-472.

18. Hodson C, Newcomb MD, Locke TF, et al. Childhood adversity, poly-substance use, and disordered eating in adolescent Latinas: mediated and indirect paths in a community sample. Child Abuse Negl. 2006;30(9):1017-1036.

19. Wills TA, Walker C, Mendoza D, et al. Behavioral and emotional selfcontrol: relations to substance use in samples of middle and high school students. Psychol Addict Behav. 2006;20(3):265-227.

20. Santrock JW. Child development. 11th ed. New York: McGraw-Hill; 2013. p. 1-656.

21. Walters GD. Addiction and identity: Exploring the possibility of a relationship. Psychology of Addictive Behaviors. 1996;10(1):9-17.

22. Muehlenkamp JJ. Self-injurious behavior as a separate clinical syndrome. Am J Orthopsychiatry. 2005;75(2):324-333.

23. Marlatt GA, Donovan DM. Relapse Prevention: Maintenance Strategies in the Treatment of Addictive Behaviors. 2nd ed. New York: Guilford Press; 2005. p. 1-416.

24. Best D, Gow J, Knox T, et al. Mapping the recovery stories of drinkers and drug users in Glasgow: quality of life and its associations with measures of recovery capital. Drug Alcohol Rev. 2012;31(3):334-341.

25. Capuzzi D, Stauffer MD. Foundations of Addictions Counseling. Pearson Publishing, Upper Saddle River, NJ, USA; 2012. p. 1-448.

26. Longabaugh R, Wirtz PW, Zweben A, et al. Network support for drinking, Alcoholics Anonymous and long-term matching effects. Addiction. 1998;93(9):1313-1333.

27. Donovan DM. Efficacy and effectiveness: complementary findings from two multisite trials evaluating outcomes of alcohol treatments differing in theoretical orientations. Alcohol Clin Exp Res. 1999;23(3):564-572.

28. World Health Organization. Risks to Mental Health: An Overview of Vulnerabilities and Risk Factors. WHO, Geneva. 2012.

29. http://www.drugabuse.gov/related-topics/addiction-science

30. Bryant AL, Zimmerman MA. Examining the effects of academic beliefs and behaviors on changes in substance use among urban adolescents. Journal of Educational Psychology. 2002;94(3):621-637.

31. Griffin KW, Botvin GJ, Scheier LM, et al. Parenting practices as predictors of substance use, delinquency, and aggression among urban minority youth: moderating effects of family structure and gender. Psychol Addict Behav. 2000;14(2):174-184.

32. Wills TA, Gibbon FX, Gerrard M, et al. Protection and vulnerability processes relevant for early onset of substance use: A test among African American children. Health Psychology. 2000;19(3):253-263.

33. Allen JP, Hauser ST, Borman-Spurrell E. Attachment theory as a framework for understanding sequelae of severe adolescent psychopathology: An 11-year follow-up study. J Consult Clin Psychol. 1996;64(2):254-263.

34. Newcomb MD, Scheie LM, Bentle PM. Effects of adolescent drug use on adult mental health: A prospective study of a community sample. Experimental \& Clinical Psychopharmacology. 1993;1(1-4):215-241. 
35. Ridenour TA, Cottler LB, Robins LN, et al. Test of the plausibility of adolescent substance use playing a causal role in developing adulthood antisocial behavior. J Abnorm Psychol. 2002;111(1):144-155.

36. Crowell SE, Beauchaine TP, Linehan MM. A biosocial developmental model of borderline personality: Elaborating and extending Linehan's theory. Psychol Bull. 2009;135(3):495-510.

37. Rathus JH, Miller AL. Dialectical behavior therapy adapted for suicidal adolescents. Suicide Life Threat Behav. 2002;32(2):146-57.

38. Katz LY, Cox BJ, Gunasekara S, et al. Feasibility of dialectical behavior therapy for suicidal adolescent inpatients. $\mathrm{J} \mathrm{Am} \mathrm{Acad} \mathrm{Child} \mathrm{Adolesc}$ Psychiatry. 2004;4(3):276-282.
39. Stotts AL, Northrup TF. The promise of third-wave behavioral therapies in the treatment of substance use disorders. Current Opinion in Psychology. 2015;2:75-81.

40. McLellan AT, Lewis DC, O'Brien CP, et al. Drug dependence, a chronic medical illness: implications for treatment, insurance, and outcomes evaluation. JAMA. 2000;284(13):1689-1695.

41. Center for Substance Abuse Treatment. Substance Abuse Treatment for Persons with Co-Occurring Disorders. Substance Abuse and Mental Health Services Administration. Rockville, MD, USA, 2005 